\title{
Thermally superactive artificial kagome spin ice structures obtained with the interfacial Dzyaloshinskii-Moriya interaction
}

\author{
Kevin Hofhuis $\odot,{ }^{1,2,{ }^{*}}$ Aleš Hrabec, ${ }^{1,2,3}$ Hanu Arava $\odot,{ }^{1,2}$ Naëmi Leo $\odot,{ }^{1,2}$ Yen-Lin Huang, ${ }^{4}$ Rajesh V. Chopdekar $\odot,{ }^{5}$ \\ Sergii Parchenko, ${ }^{1,2,6}$ Armin Kleibert, ${ }^{6}$ Sabri Koraltan, ${ }^{7}$ Claas Abert, ${ }^{7,8}$ Christoph Vogler, ${ }^{7}$ Dieter Suess, ${ }^{7,8}$ \\ Peter M. Derlet, ${ }^{1,9}$ and Laura J. Heyderman ${ }^{1,2, \dagger}$ \\ ${ }^{1}$ Laboratory for Mesoscopic Systems, Department of Materials, ETH Zurich, 8093 Zurich, Switzerland \\ ${ }^{2}$ Laboratory for Multiscale Materials Experiments, Paul Scherrer Institute, 5232 Villigen PSI, Switzerland \\ ${ }^{3}$ Laboratory for Magnetism and Interface Physics, Department of Materials, ETH Zurich, 8093 Zurich, Switzerland \\ ${ }^{4}$ Department of Materials Science and Engineering, University of California, Berkeley, California 94720, USA \\ ${ }^{5}$ Advanced Light Source, Lawrence Berkeley National Laboratory (LBNL), 1 Cyclotron Road, Berkeley, California 94720, USA \\ ${ }^{6}$ Swiss Light Source (SLS), Paul Scherrer Institute, 5232 Villigen PSI, Switzerland \\ ${ }^{7}$ Faculty of Physics, University of Vienna, Vienna, Austria \\ ${ }^{8}$ University of Vienna Research Platform MMM Mathematics - Magnetism - Materials, University of Vienna, Austria \\ ${ }^{9}$ Condensed Matter Theory Group, Paul Scherrer Institute, 5232 Villigen PSI, Switzerland
}

(Received 19 February 2020; revised 5 October 2020; accepted 11 October 2020; published 9 November 2020)

\begin{abstract}
Artificial kagome spin ice exhibits exotic magnetic correlations driven by a combination of geometric frustration and dipolar interactions that, at low-enough temperature, can result in ordered phases. This order, whether it is the ground state of several kagome rings, or the theoretically predicted long-range order of an extended array, has yet to be experimentally observed. By introducing an interfacial Dzyaloshinskii-Moriya interaction, we are able to reduce the blocking temperature of the individual nanomagnets, allowing a system of 30 kagome nanomagnets to explore its vast manifold of microstates and find its ground state. Furthermore, the extracted magnetic correlations in an extended artificial kagome spin ice are found to exhibit quantitative signatures of long-range charge order, providing evidence of the theoretically predicted continuous phase transition to the charge-ordered state. The significant lowering of the blocking temperature in nanomagnets is important for the exploitation of superparamagnetism in artificial spin systems and devices.
\end{abstract}

DOI: 10.1103/PhysRevB.102.180405

Artificial spin ices are lithographically defined magnetic metamaterials that are used to explore emergent magnetic phenomena arising from geometrical frustration [1-3]. These single-domain bistable nanomagnets are arranged on twodimensional lattices and, as a result of geometrical frustration, not all pairwise magnetic interactions can be optimized at the same time. A prominent example is the artificial kagome spin ice [4-6], where a number of phases have been theoretically predicted $[7,8]$. On decreasing the temperature within the icerule sector, a transition to a vertex-charge ordered phase is predicted to occur at $T_{\text {crit }}^{\text {charge }}=0.35 J_{\mathrm{NN}}$, where $J_{\mathrm{NN}} \propto \mathrm{m}^{2} / a^{3}$ is the nearest-neighbor coupling strength between nanomagnets of magnetic moment $m=|\boldsymbol{m}|$ separated by a distance $a$ (see Fig. 1). As the temperature is further decreased to $T_{\text {crit }}^{\text {spin }} \approx 0.12 J_{\mathrm{NN}}$, the kagome spin ice is predicted to transition into a long-range spin- and charge-ordered ground state.

In contrast to artificial square ice, which undergoes an Ising transition directly from the paramagnetic state at the critical temperature $T_{\text {crit }} \sim 2.5 J_{\mathrm{NN}}$ [9], the kagome system has been found to freeze at its blocking temperature before such a transition can be observed. In fact, with spatially resolved

\footnotetext{
*khofhuis@gmail.com

†laura.heyderman@psi.ch
}

measurements of thermally active artificial kagome spin ices, only ice-rule obedience [10] and the formation of vertexcharge crystallites [11-13] have so far been observed. Neither the low-temperature equilibrium correlations associated with the vertex-charge ordering $\left(T<T_{\text {crit }}^{\text {charge }}\right)$ nor the spin ordering $\left(T<T_{\text {crit }}^{\text {spin }}\right)$ of an extended kagome array have been observed. Even for a much smaller system consisting of seven hexagonal rings, as shown in Fig. 1, the probability of obtaining one of the two ground state configurations is small, since there are $10^{5}$ competing low-energy microstates that satisfy the ice rule. Indeed, to date, the ground state of such seven-ring structures has only been achieved by direct writing [14], but not using thermally active nanomagnets [15].

To observe dynamics at the temperature scales of $T_{\text {crit }}^{\text {charge }}$ or $T_{\text {crit }}^{\text {spin }}$, the blocking temperature defined as $T_{\mathrm{B}}=E_{\mathrm{B}} /\left(k_{\mathrm{B}} \ln \frac{\tau_{m}}{\tau_{0}}\right)$ must be reduced. Here $\tau_{m}, \tau_{0}$, and $E_{\mathrm{B}}$ are the characteristic timescale of the experimental measurement, the inverse attempt frequency of magnetic reorientation [16], and the barrier energy for magnetic reorientation, respectively, and $k_{\mathrm{B}}$ is the Boltzmann constant. For nanomagnets, $E_{\mathrm{B}}=K_{\mathrm{eff}} V$, where $K_{\text {eff }}$ is the effective local magnetic anisotropy, and $V$ is the volume of the nanomagnet given by its length $L$, width $W$, and thickness $h$ (see Fig. 1). Thus $T_{\mathrm{B}}$ may be reduced by decreasing the volume of the nanomagnets. However, since the coupling constant $J_{\mathrm{NN}} \propto m^{2}$ and $m=M_{\mathrm{sat}} V$, the critical 

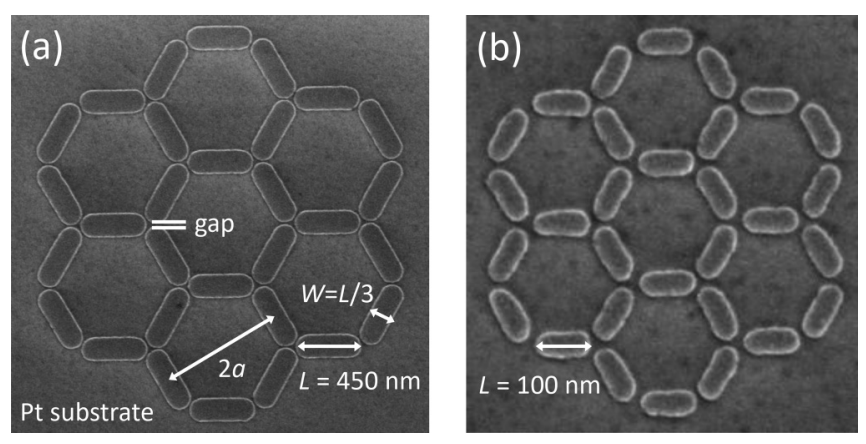

FIG. 1. Seven-ring artificial kagome spin ice structures. Scanning electron microscope images of seven-ring artificial kagome structures patterned on a $5 \mathrm{~nm}$ Pt film with $30 \mathrm{Ni}_{80} \mathrm{Fe}_{20}$ nanomagnets of dimensions length $L \times$ width $W \times$ thickness $h$ of (a) $450 \mathrm{~nm} \times$ $150 \mathrm{~nm} \times 2.7 \mathrm{~nm}$ and (b) $100 \mathrm{~nm} \times 34 \mathrm{~nm} \times 3.3 \mathrm{~nm}$. The lattice parameter $a$ is defined by $L$ and a gap of $20 \mathrm{~nm}$.

temperatures will also reduce and by a far greater proportion. This latter dependency has been exploited to produce equilibrium room temperature dynamics by either decreasing the nanomagnet volume $V[9,17]$ or the saturation magnetization $M_{\text {sat }}[18,19]$. However, with the ratio $T_{\mathrm{B}} / T_{\text {crit }}$ scaling as $a^{3} / V[\propto L / h$; see Secs. I and II of the Supplemental Material (SM) [20]], such an approach, while useful for the spin ice regime $\left(T \gg T_{\text {crit }}^{\text {charge }}\right)$, is not an appropriate strategy for observing equilibrium dynamics at $T<T_{\text {crit }}^{\text {charge }}$.

In this work, we employ an approach to decrease the nanomagnet blocking temperature $T_{\mathrm{B}}$ by exploiting the interfacial Dzyaloshinskii-Moriya interaction (DMI) [21,22]. This leads to the lowering of the effective anisotropy constant $K_{\text {eff }}$ given by the shape anisotropy $K_{x y}$ and a countering effective DMI anisotropy $K_{\text {DMI }}$ [23]. The interfacial DMI therefore lowers $T_{\mathrm{B}}$, but not $T_{\text {crit }}$, as the dipolar interactions between nanomagnets remain unchanged. As has been shown by Cubukcu et al. [23], a sufficiently strong DMI will modify the orientation of the easy axis originally dictated by shape anisotropy. We exploit this strategy, introducing interfacial DMI to produce thermally superactive nanomagnets with rapidly fluctuating moments down to temperatures of $T=$ $50 \mathrm{~K}$. Arrays of such superactive nanomagnets can efficiently explore the vast network of microstates of the kagome spin ice, which we demonstrate with the observation of ground state ordering in seven-ring structures, as well as correlations close to the charge order transition in extended kagome lattices.

As indicated by the results of micromagnetic simulations, shown in Fig. 2(a) and the Supplemental Material movies [20], a quasicoherent rotation governs the magnetization reversal as determined by minimum energy path simulations [24-26] using bulk $\mathrm{Ni}_{80} \mathrm{Fe}_{20}$ parameters [27]. The energy associated with the intermediate state sets an energy barrier $E_{\mathrm{B}}$ with the net magnetization momentarily pointing along the short axis of the nanomagnet. The reduction in anisotropy as a result of introducing DMI originates from an out-of-plane canting of the spins at the nanomagnet edges $[23,28,29]$. This decreases the local spin-spin energy, as indicated by the areas highlighted in red in Fig. 2(a), and interestingly results in a more coherent reversal upon the (a)
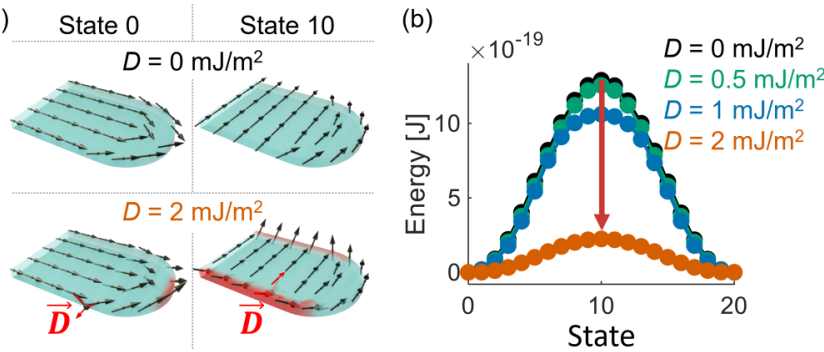

FIG. 2. Minimum energy path simulation of the moment reversal in the presence of the interfacial DMI. Simulated spin structures of a nanomagnet in its stable state (State 0 , with magnetization along the long axis) and intermediate states at the energy barrier (State $10)$, both with and without interfacial DMI $(D)$. With the interfacial DMI $\left(D=2.0 \mathrm{~mJ} / \mathrm{m}^{2}\right)$, the tilt of the spin at the edges, highlighted in red, reduces the total energy during magnetization reversal. (b) The minimum energy path during magnetization reversal is simulated using a full micromagnetic model with four nearest neighbors of nanomagnets and variable DMI strength $D$. The energy barrier $E_{\mathrm{B}}$ is the difference in energy between State 0 and State 10 .

addition of the interfacial DMI. The energy barrier as a function of DMI strength $D$ is given in Fig. 2(b), where stronger DMI leads to a larger reduction in the energy barrier as highlighted by the red arrow. We furthermore provide a direct comparison of blocking temperatures of nanomagnets fabricated on Pt (with DMI) and Si (without DMI) using temperature-dependent $\mathrm{x}$-ray photoemission electron microscopy (X-PEEM) [30] (see SM Fig. 2 [20]).

The interfacial DMI effect entails a more complex dependence of the blocking temperature on the nanomagnetic volume, which in turn can be exploited to reduce $T_{\mathrm{B}} / T_{\text {crit }} \propto$ $T_{\mathrm{B}} / J_{\mathrm{NN}}$. The relation, as derived in the Supplemental Material [20],

$$
\frac{T_{\mathrm{B}}}{J_{\mathrm{NN}}} \propto\left(K_{x y} \frac{L}{h}-\frac{2 D_{s}^{2}}{\sqrt{A_{\mathrm{ex}} K_{x z}}} \frac{1}{h^{3}}\right),
$$

indicates that there exists a maximum $T_{\mathrm{B}} / J_{\mathrm{NN}} \propto L^{3 / 2} / D_{s}$ at $h_{\max } \propto 1 / \sqrt{L}$. For $h$ lower than this value, $T_{\mathrm{B}} / J_{\mathrm{NN}} \mathrm{de}-$ creases due to the increasing negative DMI contribution (DMI-dominant regime), whereas above this value a decrease is due to a decrease in the shape anisotropy contribution. The maximum $h_{\max }$ separating the two regimes may be decreased by either lowering the lateral dimension $L$, or by increasing the effective DMI $D$ by reducing the thickness $h$ [31]. Given that the above expression is for an ideal system, and that experimental realizations have increasing disorder as the dimensions of the nanomagnets are reduced, we investigate the predicted DMI dependence by exploring a range of seven-ring kagome systems in which both $L$ and $h$ are varied.

The seven-ring kagome structures are fabricated using $\mathrm{Ni}_{80} \mathrm{Fe}_{20}$ nanomagnets with lengths $L=450,300,210,150$, and $100 \mathrm{~nm}$, and a fixed aspect ratio $L / W=3$. The samples were fabricated with lift-off assisted electron beam lithography from a $\mathrm{Ni}_{80} \mathrm{Fe}_{20}$ thin-film wedge of variable thickness $h$ between 0 and $7 \mathrm{~nm}$ over a distance of $6 \mathrm{~mm}$ deposited by thermal evaporation (base pressure $10^{-7} \mathrm{mbar}$ ). The gaps between neighboring nanomagnets [see Fig. 1(a)] are chosen to be as small as lithographically possible (here $20 \mathrm{~nm}$ ) to 

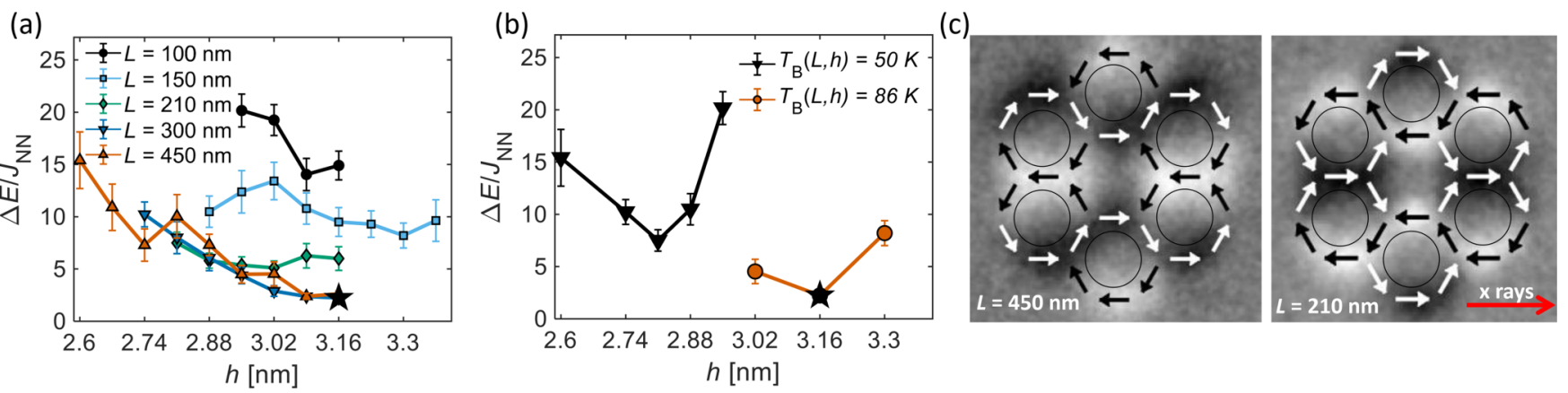

FIG. 3. Magnetic ordering in seven-ring kagome structures. (a) Normalized point-dipolar energy of magnetic configurations compared to the ground state $\left[\Delta E / J_{\mathrm{NN}}=\left\langle E_{\mathrm{config}}-E_{\mathrm{GS}}\right\rangle / J_{\mathrm{NN}}\right]$ determined from X-PEEM images, where each point corresponds to a kagome structure incorporating nanomagnets with a particular $L$ and $h$. For each data point, the configuration energies of 20 seven-ring structures are averaged, with the error bars indicating the standard deviation. The structure with $\Delta E / J_{\mathrm{NN}}$ closest to the ground state is indicated with a black star. (b) Normalized configuration energies $\Delta E / J_{\mathrm{NN}}$ for two experimentally determined blocking temperature values $T_{\mathrm{B}}(L, h)$. (c) Example XPEEM images of seven-ring artificial kagome spin ice structures in the ground state with nanomagnet dimensions $(L \times W \times h) 450 \mathrm{~nm} \times$ $150 \mathrm{~nm} \times 2.7 \mathrm{~nm}$ (top image) and $210 \mathrm{~nm} \times 70 \mathrm{~nm} \times 3.1 \mathrm{~nm}$ (bottom image) fabricated on top of a $5 \mathrm{~nm}$ Pt film. The arrows indicate the orientation of the magnetic moments and the circles indicate closed loops of head-to-tail moments. The red arrow indicates the $\mathrm{x}$-ray direction.

maximize $J_{\mathrm{NN}} \propto 1 / a^{3}$ by minimizing $a$. The interfacial DMI is introduced by fabricating the seven-ring structures on top of a $5 \mathrm{~nm}$ thin Pt film. From the systematic analysis described in the Supplemental Material [20], the chosen nanomagnet dimensions are expected to result in a regime that is dominated by DMI and therefore a reduction in blocking temperature.

Arrays of such seven-ring kagome structures were exposed to a quenching protocol in which the temperature was reduced from 300 down to $30 \mathrm{~K}$ over a period of $1 \mathrm{~h}$. Imaging of the resulting magnetic configurations was performed by means of X-PEEM [32]. Magnetic contrast is obtained by exploiting $\mathrm{X}$-ray magnetic circular dichroism (XMCD) and dividing the images obtained with right- and left-circularly polarized $\mathrm{x}$ rays at the $\mathrm{Fe} L_{3}$ edge [33]. The resulting XMCD contrast is proportional to the projection of the magnetic moments onto the x-ray propagation vector, with moments pointing towards (away from) the incoming $\mathrm{x}$ rays appearing bright (dark) with a sensitivity and resolution that allows for the determination of the entire magnetic configuration. At $30 \mathrm{~K}$, all configurations were no longer thermally active within the observational time frame of the experiment. To quantify how close the observed magnetic configurations are to the known ground state, the point-dipolar energy $E_{\text {config }}$ is determined for each seven-ring structure. Specifically, we determine the average point-dipolar energy above the ground state $\Delta E=\left\langle E_{\text {config }}-E_{\mathrm{GS}}\right\rangle$ for 20 equivalent structures, where $E_{\mathrm{GS}}$ is the point-dipolar energy of the ground state. In order to compare the energy of structures with different dimensions, $\Delta E$ is normalized to the nearestneighbor dipolar coupling pre-factor $J_{\mathrm{NN}}$.

A low value of $\Delta E$ suggests that the system remained thermally active at low temperatures, where it can explore the large number of low-energy states, thus reflecting a low blocking temperature $T_{\mathrm{B}}$. In Fig. 3(a) the obtained values of $\Delta E$ as a function of $h$ for different $L$ are displayed for 34 unique values of $h$ and $L$. The magnetic configurations closest to the ground state energy are indicated with a black star and involve $L=300 \mathrm{~nm}$ and $L=450 \mathrm{~nm}$ at $h \simeq 3.2 \mathrm{~nm}$. In total, three seven-ring ground state configurations were found, two of which are shown in Fig. 3(c).
In Fig. 3(a), it is demonstrated that samples with a larger $L$ generally exhibit a lower energy configuration (and therefore a correspondingly lower blocking temperature) with increasing $h$, a trend opposite to what is expected from Eq. (1) for the DMI-dominated regime. This observation is attributed to increasing disorder for smaller $h$, where it is known that such thin nanomagnets have a reduced magnetization [34]. Encouraging is that, by increasing $h, \Delta E$ is indeed reduced, indicating the emergence of the DMI effect and the observation of the ground state configuration at $h \simeq 3.2 \mathrm{~nm}$. For the samples with smaller $L$, the trend is not so apparent with the magnetic configurations generally having energies well above that of the ground state as well as a broader range of $\Delta E$. Thus systems with smaller nanomagnets tend to fall out of equilibrium at higher temperature, an observation that can be rationalized again by the increasing presence of disorder. Indeed, as the lateral dimensions of the nanomagnets are reduced, edge roughness, and therefore pinning, becomes more important. This in turn increases $T_{\mathrm{B}}$, so that the system falls out of equilibrium at higher temperatures. This has also been observed in connected systems where lithographic irregularities for smaller sizes adversely affect the magnetic ordering $[35,36]$ and is further discussed in the Supplemental Material [20].

The presence of disorder thus complicates the relationship between $\Delta E$ and the blocking temperature. To quantify this, we define an upper estimate to $T_{\mathrm{B}}$ as the temperature below which no more than three to five moment fluctuations were observed within $50 \mathrm{~s}$, corresponding to the time of ten consecutive XMCD images. We thus obtain $T_{\mathrm{B}}$ as a function of $L$ and $h$ [see SM Fig. 4(a) [20]], and in Fig. 3(b) plot the data from (a) that have a $T_{\mathrm{B}}$ of $50 \pm 5 \mathrm{~K}$ and $86 \pm 5 \mathrm{~K}$. Inspection of the figure reveals a nonmonotonic relationship between $\Delta E$ and $T_{\mathrm{B}}$, characterized by a minimum in $\Delta E$. An optimal geometry exists that involves the minimization of microscopic disorder in terms of $h$ and lithographic irregularities in terms of $L$, to give an artificial kagome spin ice whose low-energy configurations, and indeed the ground state, in smaller structures can be accessed-with a 
characteristic blocking temperature controlled by DMI through Eq. (1).

We now turn our attention to the magnetic moment and charge ordering of an extended artificial kagome spin ice consisting of 3684 nanomagnets arranged on the sites of a kagome lattice, which has been cooled to $30 \mathrm{~K}$ using the same procedure described above. With the nanomagnet dimension being $450 \mathrm{~nm} \times 150 \mathrm{~nm} \times 2.9 \mathrm{~nm}$, the equivalent upper estimate of the blocking temperature in the seven-ring structure would be $T_{\mathrm{B}}=80 \pm 5 \mathrm{~K}$ [Fig. 3(b)]. These dimensions are comparable to those used in previous systems without DMI [10], which allows us to directly compare the degree of ordering in artificial kagome spin ice systems with and without the interfacial DMI. For this earlier artificial kagome system, the upper estimate of the blocking temperature using the same criterion as chosen here was found to be $T_{\mathrm{B}}=420 \mathrm{~K}$ [10].

An alternative approach to estimating the absolute blocking temperature is to deduce the reduced blocking temperature $T_{\text {red }}^{D \neq 0}=T / J_{\mathrm{NN}}$ at which the extended system freezes, therefore giving insight into what part of the phase diagram can be probed under equilibrium conditions. The value of $T_{\text {red }}$ can be obtained from the spin-spin and charge-charge correlators extracted from the XMCD images that are in turn compared to those obtained from Monte Carlo simulations of the point-dipolar interacting kagome system. The temperaturedependent values of the simulated spin and charge correlators $C_{i j}$ are shown in Figs. 4(a) and 4(b), respectively. The reduced temperature for the experimentally obtained correlator values (filled circles, with DMI) is determined with a standard-deviation correlator analysis using a spread-out function $[13,37]$ to be $T_{\text {red }}^{D \neq 0}=0.84$ and is indicated by the left-hand dashed line in Fig. 4.

We find our thermally superactive artificial kagome spin ice to be close to the predicted transition to the charge-ordered phase at $T_{\text {crit }}^{\text {charge }} / J_{\mathrm{NN}} \approx 0.35$ (see SM Fig. 5 [20]). Performing the same spin and charge correlator analysis (open circles in Fig. 4 indicated by the right-hand dashed line) on the artificial kagome spin ice without interfacial DMI measured by Farhan et al. [10], gives $T_{\text {red }}^{D=0}=4.49$.

The observation that the experimentally obtained temperatures $T_{\mathrm{B}}$ and $T_{\text {red }}$ for the artificial kagome spin ice with DMI ( $80 \mathrm{~K}$ and 0.84$)$ and without DMI (420 K and 4.49) are both decreased by a factor of 5 , indicates a consistency in our approach in which the dipolar coupling strength $J_{\mathrm{NN}}$ is not significantly affected by interfacial DMI. We can therefore conclude that DMI is responsible for a considerable decrease in blocking temperature, thereby extending the experimentally accessible equilibrium temperature range of the artificial kagome system. The present work therefore provides a new way to control the $T_{\mathrm{B}}$ of thermally active nanomagnets, exploring the disorder-induced limits of downsizing their lateral dimensions and thickness, which remains an obstacle to observe the low-temperature phases of artificial kagome spin ice.

In conclusion, we demonstrated that the introduction of the interfacial DMI leads to a reduction in the effective anisotropy of nanomagnets, thus enhancing the superparamagnetic fluctuation rate while maintaining dipolar interactions between the nanomagnets. We demonstrated ground state ordering in seven-ring kagome structures, and obtained a thermally superactive system with a blocking temperature very close to the

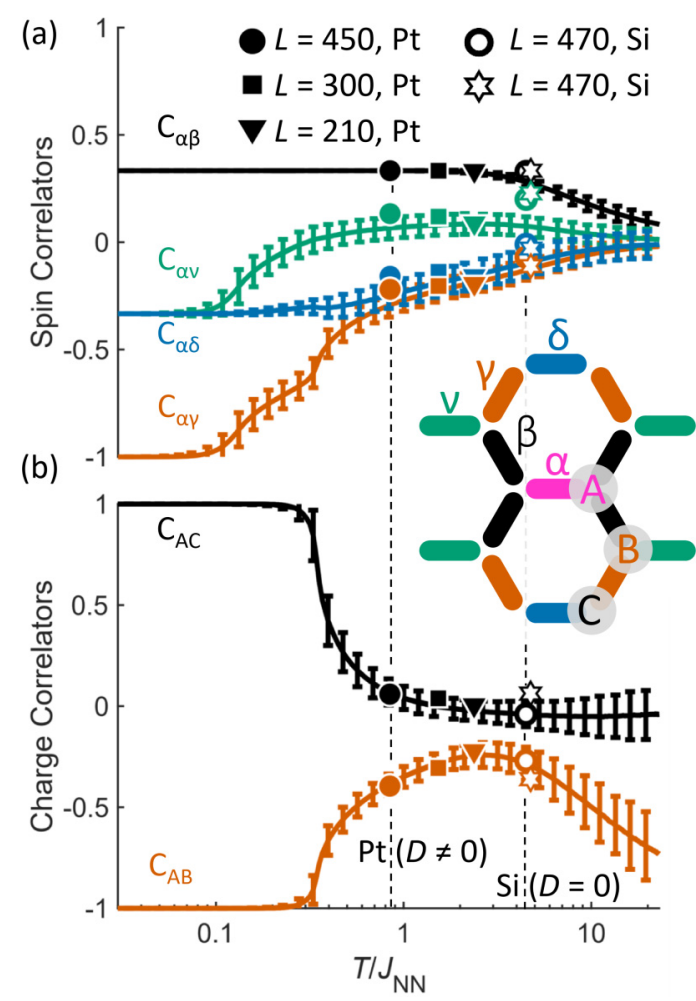

FIG. 4. Spatial correlations in artificial kagome spin ice. Monte Carlo simulations of (a) spin-spin correlations and (b) charge-charge correlations, as indicated in the schematic of the artificial kagome spin ice. The first four neighboring nanomagnets to $\alpha$ are indicated with $\beta, \gamma, v$, and $\delta$, while the first two neighboring vertex charges to $A$ are indicated with $B$ and $C$. The charges come from the vertex containing two-in/one-out or two-out/one-in in moments resulting in $\pm 1 q$ charge [6]. The filled markers denote the correlation values determined from the X-PEEM images for an artificial kagome spin ice with interfacial DMI. The open markers show the correlators obtained for a system without the interfacial DMI, with the open circles representing the values from Farhan et al. [10]. The dashed lines indicate the two systems with similar $J_{\mathrm{NN}}$ with and without DMI.

charge-order transition in an extended artificial kagome spin ice. The implementation of these superactive nanomagnets will facilitate future studies in other highly frustrated artificial spin systems [38-43]. In addition, the ability to lower the energy barrier for switching, while maintaining a considerable coupling strength, will lead to enhanced reliability and reduced operational power for computation with nanomagnets [44].

The dataset that supports this study is available via the Zenodo repository [45].

We thank Stanislas Rohart, Sebastian Gliga, and Olle Heinonen for initial discussions, and Alan Farhan for his support and discussions. This work was supported by the Swiss National Science Foundation (Project 200020_172774). A.H. was funded by the European Union's Horizon 2020 research and innovation program under Marie Skłodowska-Curie Grant Agreement No. 794207 (ASIQS). N.L. has received funding 
from the European Union's Horizon 2020 research and innovation programme under the Marie Słodowska Curie Grant Agreement No. 844304 (LICONAMCO). S.K., C.A. and D.S. acknowledge the support from Austrian Science Fund by project $\mathrm{P}$ 33748. The computational results presented have been achieved, in part, using the Vienna Scientific Cluster
(VSC). Part of this research used resources of the PEEM3 Beamline (11.0.1) at the Advanced Light Source, a DOE Office of Science User Facility under Contract No. DE-AC0205CH11231 and at the Surface/Interface: Microscopy (SIM) beamline of the Swiss Light Source, Paul Scherrer Institute, Villigen PSI, Switzerland.
[1] A. R. Fert, Mater. Sci. Forum 59, 439 (1990).

[2] N. Rougemaille and B. Canals, Eur. Phys. J. B 92, 62 (2019).

[3] S. H. Skjærvø, C. H. Marrows, R. L. Stamps, and L. J. Heyderman, Nat. Rev. Phys. 2, 13 (2020).

[4] S. Ladak, D. E. Read, G. K. Perkins, L. F. Cohen, and W. R. Branford, Nat. Phys. 6, 359 (2010).

[5] N. Rougemaille, F. Montaigne, B. Canals, A. Duluard, D. Lacour, M. Hehn, R. Belkhou, O. Fruchart, S. El Moussaoui, A. Bendounan, and F. Maccherozzi, Phys. Rev. Lett. 106, 057209 (2011).

[6] E. Mengotti, L. J. Heyderman, A. F. Rodríguez, F. Nolting, R. V. Hügli, and H.-B. Braun, Nat. Phys. 7, 68 (2011).

[7] G. Möller and R. Moessner, Phys. Rev. B 80, 140409(R) (2009).

[8] G.-W. Chern, P. Mellado, and O. Tchernyshyov, Phys. Rev. Lett. 106, 207202 (2011).

[9] O. Sendetskyi, V. Scagnoli, N. Leo, L. Anghinolfi, A. Alberca, J. Lüning, U. Staub, P. M. Derlet, and L. J. Heyderman, Phys. Rev. B 99, 214430 (2019).

[10] A. Farhan, P. M. Derlet, L. Anghinolfi, A. Kleibert, and L. J. Heyderman, Phys. Rev. B 96, 064409 (2017).

[11] S. Zhang, I. Gilbert, C. Nisoli, G.-W. Chern, M. J. Erickson, L. O'Brien, C. Leighton, P. E. Lammert, V. H. Crespi, and P. Schiffer, Nature (London) 500, 553 (2013).

[12] M. E. Brooks-Bartlett, S. T. Banks, L. D. C. Jaubert, A. Harman-Clarke, and P. C. W. Holdsworth, Phys. Rev. X 4, 011007 (2014).

[13] B. Canals, I.-A. Chioar, V.-D. Nguyen, M. Hehn, D. Lacour, F. Montaigne, A. Locatelli, T. O. Menteş, B. S. Burgos, and N. Rougemaille, Nat. Commun. 7, 11446 (2016).

[14] J. C. Gartside, D. M. Arroo, D. M. Burn, V. L. Bemmer, A. Moskalenko, L. F. Cohen, and W. R. Branford, Nat. Nanotechnol. 13, 53 (2018).

[15] A. Farhan, A. Kleibert, P. M. Derlet, L. Anghinolfi, A. Balan, R. V. Chopdekar, M. Wyss, S. Gliga, F. Nolting, and L. J. Heyderman, Phys. Rev. B 89, 214405 (2014).

[16] S. Y. Liashko, H. Jónsson, and V. M. Uzdin, New J. Phys. 19, 113008 (2017).

[17] A. Farhan, P. M. Derlet, A. Kleibert, A. Balan, R. V. Chopdekar, M. Wyss, L. Anghinolfi, F. Nolting, and L. J. Heyderman, Nat. Phys. 9, 375 (2013).

[18] V. Kapaklis, U. B. Arnalds, A. Farhan, R. V. Chopdekar, A. Balan, A. Scholl, L. J. Heyderman, and B. Hjörvarsson, Nat. Nanotechnol. 9, 514 (2014).

[19] S. A. Morley, S. T. Riley, J.-M. Porro, M. C. Rosamond, E. H. Linfield, J. E. Cunningham, S. Langridge, and C. H. Marrows, Sci. Rep. 8, 4750 (2018).

[20] See Supplemental Material at http://link.aps.org/supplemental/ 10.1103/PhysRevB.102.180405 for supplementary animations of the magnetization reversal with and without DMI and sup- plemental X-PEEM measurements, derivation of Eq. (1), and simulations.

[21] A. R. Fert, in Materials Science Forum, Vol. 59 (Trans Tech Publ, 1990), pp. 439-480.

[22] A. Crépieux and C. Lacroix, J. Magn. Magn. Mater. 182, 341 (1998).

[23] M. Cubukcu, J. Sampaio, K. Bouzehouane, D. Apalkov, A. V. Khvalkovskiy, V. Cros, and N. Reyren, Phys. Rev. B 93, 020401(R) (2016).

[24] W. E, W. Ren, and E. Vanden-Eijnden, J. Chem. Phys. 126, 164103 (2007).

[25] C. Abert, Eur. Phys. J. B 92, 120 (2019).

[26] S. Koraltan, M. Pancaldi, N. Leo, C. Abert, C. Vogler, K. Hofhuis, F. Slanovc, F. Bruckner, P. Heistracher, M. Menniti, P. Vavassori, and D. Suess, Phys. Rev. B 102, 064410 (2020).

[27] R. C. O'Handley, Modern Magnetic Materials: Principles and Applications (Wiley, New York, 2000), p. 768.

[28] S. Rohart and A. Thiaville, Phys. Rev. B 88, 184422 (2013).

[29] J. Sampaio, A. V. Khvalkovskiy, M. Kuteifan, M. Cubukcu, D. Apalkov, V. Lomakin, V. Cros, and N. Reyren, Appl. Phys. Lett. 108, 112403 (2016).

[30] L. Le Guyader, A. Kleibert, A. Fraile Rodríguez, S. El Moussaoui, A. Balan, M. Buzzi, J. Raabe, and F. Nolting, J. Electron Spectrosc. Relat. Phenom. 185, 371 (2012).

[31] H. T. Nembach, J. M. Shaw, M. Weiler, E. Jué, and T. J. Silva, Nat. Phys. 11, 825 (2015).

[32] A. Doran, M. Church, T. Miller, G. Morrison, A. T. Young, and A. Scholl, J. Electron Spectrosc. Relat. Phenom. 185, 340 (2012).

[33] J. Stöhr, Y. Wu, B. D. Hermsmeier, M. G. Samant, G. R. Harp, S. Koranda, D. Dunham, and B. P. Tonner, Science 259, 658 (1993).

[34] X. Zhang, Y. Lao, J. Sklenar, N. S. Bingham, J. T. Batley, J. D. Watts, C. Nisoli, C. Leighton, and P. Schiffer, APL Mater. 7, 111112 (2019).

[35] Z. Budrikis, J. P. Morgan, J. Akerman, A. Stein, P. Politi, S. Langridge, C. H. Marrows, and R. L. Stamps, Phys. Rev. Lett. 109, 037203 (2012).

[36] J. Drisko, S. Daunheimer, and J. Cumings, Phys. Rev. B 91, 224406 (2015).

[37] I. A. Chioar, B. Canals, D. Lacour, M. Hehn, B. Santos Burgos, T. O. Menteş, A. Locatelli, F. Montaigne, and N. Rougemaille, Phys. Rev. B 90, 220407(R) (2014).

[38] Y. Perrin, B. Canals, and N. Rougemaille, Nature (London) 540, 410 (2016).

[39] I. Gilbert, Y. Lao, I. Carrasquillo, L. O’Brien, J. D. Watts, M. Manno, C. Leighton, A. Scholl, C. Nisoli, and P. Schiffer, Nat. Phys. 12, 162 (2016). 
[40] D. Shi, Z. Budrikis, A. Stein, S. A. Morley, P. D. Olmsted, G. Burnell, and C. H. Marrows, Nat. Phys. 14, 309 (2018).

[41] C. Nisoli, V. Kapaklis, and P. Schiffer, Nat. Phys. 13, 200 (2017).

[42] A. Farhan, M. Saccone, C. F. Petersen, S. Dhuey, R. V. Chopdekar, Y.-L. Huang, N. Kent, Z. Chen, M. J. Alava, T. Lippert, A. Scholl, and S. v. Dijken, Sci. Adv. 5, eaav6380 (2019).
[43] M. Saccone, K. Hofhuis, D. Bracher, A. Kleibert, S. v. Dijken, and A. Farhan, Nanoscale 12, 189 (2019).

[44] H. Arava, P. M. Derlet, J. Vijayakumar, J. Cui, N. S. Bingham, A. Kleibert, and L. J. Heyderman, Nanotechnology 29, 265205 (2018).

[45] K. Hofhuis, dataset for "Thermally superactive artificial kagome spin ice structures obtained with the interfacial Dzyaloshinskii-Moriya interaction," (2020), doi: 10.5281/zenodo.4090185. 